\title{
Sodium restriction and blood pressure in hypertensive type II diabetics: randomised blind controlled and crossover studies of moderate sodium restriction and sodium supplementation
}

\author{
P M Dodson, M Beevers, R Hallworth, M J Webberley, R F Fletcher, K G Taylor
}

\begin{abstract}
Objective-To determine the effect of moderate dietary sodium restriction on the hypertension of non-insulin-dependent (type II) diabetes.

Design-Randomised parallel controlled study of moderate sodium restriction for three months compared with usual diabetic diet, followed by randomised double blind crossover trial of sustained release preparation of sodium for one month versus placebo for one month in patients continuing with sodium restriction.
\end{abstract}

Setting-Patients attending diabetic outpatient clinic of city hospital.

Patients-Thirty four patients with established type II diabetes complicated by mild hypertension (systolic blood pressure $>160 \mathrm{~mm} \mathrm{Hg}$ or diastolic pressure $>95 \mathrm{~mm} \mathrm{Hg}$ on three consecutive occasions). Patients already taking antihypertensive agents (but not diuretics) not barred from study provided that criteria for mild hypertension still met. Conditions precluding patients from study were diabetic or hypertensive nephropathy, cardiac failure, and pregnancy.

Interventions - After run in phase with recordings at seven weeks, three weeks, and time zero patients were allocated at random to receive moderate dietary sodium restriction for three months $(n=17)$ or to continue with usual diabetic diet. Subsequently nine patients in sodium restriction group continued with regimen for a further two months, during which they completed a randomised double blind crossover trial of sustained release preparation of sodium (Slow Sodium $80 \mathrm{mmol}$ daily) for one month versus matching placebo for one month.

End point-Reduction in blood pressure in type II diabetics with mild hypertension.

Measurements and main results-Supine and erect blood pressure, body weight, and 24 hour urinary sodium and potassium excretion measured monthly during parallel group and double blind crossover studies. After parallel group study sodium restriction group showed significant reduction in systolic blood pressure (supine $19.2 \mathrm{~mm} \mathrm{Hg}$, erect $21.4 \mathrm{~mm} \mathrm{Hg} ; \mathrm{p}<0.001$ ) and mean daily urinary sodium excretion (mean reduction $60 \mathrm{mmol} / 24 \mathrm{~h}$ ). There were no appreciable changes in weight, diabetic control, or diastolic pressure. No significant changes occurred in controls. In double blind crossover study mean supine systolic blood pressure rose significantly $(\mathbf{p}<\mathbf{0 . 0 0 5})$ during sodium supplementation (to $171 \mathrm{~mm} \mathrm{Hg}$ ) compared with value after three months of sodium restriction alone $(159.9 \mathrm{~mm} \mathrm{Hg})$ and after one month of placebo $(161.8 \mathrm{~mm} \mathrm{Hg})$.

Conclusions-Moderate dietary restriction of sodium has a definite hypotensive effect, which may be useful in mild hypertension of type II diabetes.

\section{Introduction}

Hypertension is common in diabetes ${ }^{2}$ but treatment may be far from satisfactory owing to the adverse effects of antihypertensive drugs. The aetiology of hypertension associated with diabetes mellitus is unclear, but reports have suggested abnormalities of sodium handling in non-insulin-dependent (type II) disease, which have not been consistently detectable in non-diabetic patients with hypertension. They include evidence that regardless of blood pressure state total exchangeable sodium is increased by around $10 \%$ in diabetics compared with normal controls matched for age and weight. ${ }^{3+}$ This abnormality may be reversed with diuretics, which cause a highly significant reduction in blood pressure. ${ }^{+}$Furthermore, in response to sodium loading urinary sodium excretion is less than in non-diabetics, ${ }^{5}$ and preliminary data suggest that there may be an abnormality in the sodium pump of diabetic red cells.

Clinical evidence of a link between sodium and blood pressure was suggested in a controlled trial of a high fibre, low fat, low sodium diet in type II diabetic hypertensives. The patients showed a substantial hypotensive response, which appeared on multiple regression analysis to be related to the reduction in sodium intake. 'The mechanism of sodium retention may be based on the action of insulin, which has a sodium retaining action in the kidneys. ${ }^{8}$ Possibly, therefore, the hyperinsulinaemia commonly associated with type II diabetes and obesity may be important."

In contrast, with type II diabetes the hypertension associated with insulin dependent (type I) disease appears to relate well to the presence of nephropathy. ${ }^{10}$ A recent report, however, has also suggested that there is increased exchangeable body sodium in type I diabetic patients even without nephropathy."

These data support the concept that diabetics retain sodium and thus may be "sodium sensitive." We have therefore performed a trial of moderate sodium restriction alone in hypertensive type II diabetics to assess its effect on blood pressure.

\section{Patients and methods}

We studied 34 type II diabetics with mild hypertension attending the diabetic clinic at this hospital. Their clinical characteristics were typical of patients with type II disease and no patient had a past or current history of treatment with insulin.

Criteria for entry to the study were three consecutive hypertensive blood pressure readings (defined by the World Health Organisation as systolic $>160 \mathrm{~mm} \mathrm{Hg}$ or diastolic $>95 \mathrm{~mm} \mathrm{Hg}$ ) in an established diabetic. ${ }^{12}$ Patients already taking antihypertensive drugs (but not diuretics) were not barred from the study provided that they still met the criteria for hypertension. Reasons for exclusion were evidence of diabetic or hypertensive nephropathy (proteinuria or raised serum creatinine concentration), cardiac failure, and pregnancy. Informed consent was given by all patients and the study approved by the local ethical committee.

\section{PARALLEL GROUP STUDY}

After recruitment a run in phase was begun with recordings at seven weeks, three weeks, and time zero, when random allocation of patients was performed. Blood pressure was measured in the supine and erect positions (after five minutes' and two minutes' 
rest, respectively) with a Hawksley random zero sphygmomanometer. All readings were taken by a separate "blind" observer (MB), diastolic pressure being recorded at Korotkoff phase V. A standard width cuff $(14 \mathrm{~cm})$ was used when the mid-arm circumference was less than $33 \mathrm{~cm}$, but for larger circumferences a $19 \mathrm{~cm}$ cuff was used. Hypoglycaemic and antihypertensive treatment continued unchanged throughout.

At the end of the run in period patients were seen monthly for three months for measurement of blood pressure, body weight, and 24 hour urinary sodium and potassium excretion. A history of alcohol intake and smoking was recorded at the initial and final visits. Blood was taken before and after three months for estimation of urea and electrolyte concentrations, liver function values, glycosylated haemoglobin concentration, and full blood count.

Diet-All patients were interviewed by the hospital dietitian $(\mathrm{RH})$. All had previously been counselled about their diabetic diet but 31 patients were eating a low carbohydrate, high fat, low fibre diet and adding salt to cooking or at table. The dietitian randomly allocated patients either to receive advice on reducing sodium intake or to a control group. Equal time was given to both groups. The appendix summarises the dietary advice about sodium intake given to 17 patients. The advice consisted simply of no salt to be added at table or in cooking and the avoidance of heavily salted foods. The advice was similar to that currently recommended for diabetic patients with regard to salt intake and ensured that the dietary changes would be modest and achievable. No advice was given on other aspects of diet (carbohydrate, fibre, or fat intake) so as to minimise any effect of the regimen on total energy intake, weight, and diabetic control. The control group consisted of 17 patients, who were instructed to continue with their usual diet. No advice was given about salt intake, but otherwise these patients followed an identical trial protocol.

DOUBLE BLIND CROSSOVER TRIAL

At the conclusion of the three month parallel group study 13 patients in the moderate sodium restriction

TABLE I-Clinical details of type II diabetic hypertensive patients studied. Except where stated otherwise values are means (SD in parentheses)

\begin{tabular}{lcc}
\hline & $\begin{array}{c}\text { Moderate sodium } \\
\text { restriction group } \\
(\mathbf{n}=17)\end{array}$ & $\begin{array}{c}\text { Control group } \\
(\mathrm{n}=17)\end{array}$ \\
\hline Sex & $12 \mathrm{M}: 5 \mathrm{~F}$ & $11 \mathrm{M}: 6 \mathrm{~F}$ \\
Age (years) & $61 \cdot 9(7 \cdot 5)$ & $61 \cdot 1(6 \cdot 3)$ \\
\% Of ideal body weight & $126 \cdot 6(20 \cdot 2)$ & $127 \cdot 5(24 \cdot 9)$ \\
Duration of diabetes (years) & $4 \cdot 1(5 \cdot 2)$ & $5 \cdot 1(3 \cdot 4)$ \\
Duration of hypertension (years) & $4 \cdot 1(3 \cdot 4)$ & $6 \cdot 5(8 \cdot 0)$ \\
$\begin{array}{l}\text { Mean erect pre-entry blood } \\
\text { pressure (mm Hg) }\end{array}$ & $179 / 98$ & $174 / 100$ \\
$\begin{array}{l}\text { Oral treatment (No of patients): } \\
\quad \text { Hypoglycaemics }\end{array}$ & 2 & 4 \\
$\quad$ Antihypertensive drug (atenolol) & 2 & 2 \\
\hline
\end{tabular}

group agreed to continue with sodium restriction while taking part in a double blind randomised crossover trial of supplementation with a sustained release preparation of sodium (Slow Sodium $80 \mathrm{mmol} /$ day) for one month compared with a matched placebo for one month. Nine patients completed the trial, the remaining four withdrawing because of side effects (two while taking the sodium supplement, two while taking placebo). Five patients received the sodium supplement first and four the placebo. Blood pressure, weight, and 24 hour urinary electrolyte excretion were measured as before.

\section{STATISTICS}

Statistical analysis was by paired and unpaired Student's $t$ tests. Randomisation was achieved with a computerised random number program for both trials.

\section{Results}

PARALLEL GROUP STUDY

Table I shows that patients randomised to the moderate sodium restriction and control groups were well matched clinically. Table II gives the changes in systolic and diastolic blood pressure and weight in the two groups.

In the sodium restriction group there were significant reductions in mean supine and erect systolic blood pressure at three months. These falls were significantly greater $(p<0.03)$ than the minor, non-significant falls in the control group (differences in mean reductions between sodium restriction and control groups: erect $11.9 \mathrm{~mm} \mathrm{Hg}(95 \%$ confidence interval 3.9 to $19.0 \mathrm{~mm} \mathrm{Hg}$ ); supine $12.9 \mathrm{~mm} \mathrm{Hg}$ ( $95 \%$ confidence interval 4.3 to $21.0 \mathrm{~mm} \mathrm{Hg}$ )).

In contrast there were no significant changes in mean diastolic pressure in either group, except in the mean erect reading of the sodium restriction group at three months $(\mathrm{p}<0.05)$. This mean fall, however, was smaller than that observed in the control group (3.4 $5.7 \mathrm{~mm} \mathrm{Hg}$ ). The number of patients with normal blood pressure at three months was greater in the sodium restriction group than among the controls (table II).

The sodium restriction group showed a significant reduction in urinary sodium output at three months $(p<0.001)$, the reduction being significantly greater than the controls $(p<0.05)$ (table III). No changes were recorded in mean urinary potassium excretion, weight, or glycosylated haemoglobin concentration. Alcohol consumption and smoking habit were unchanged after three months, and mean $\gamma$-glutamyltransferase activity and mean corpuscular volume were also unchanged.

\section{DOUBLE BLIND CROSSOVER TRIAL}

Six men and three women (mean age 62 (SD 6.5) years) with a mean duration of diabetes of five years completed the randomised double blind crossover

TABLE II - Summary of clinical changes recorded in three month paratlel controlled study. Except where stated otherwise values are means (SD in parentheses)

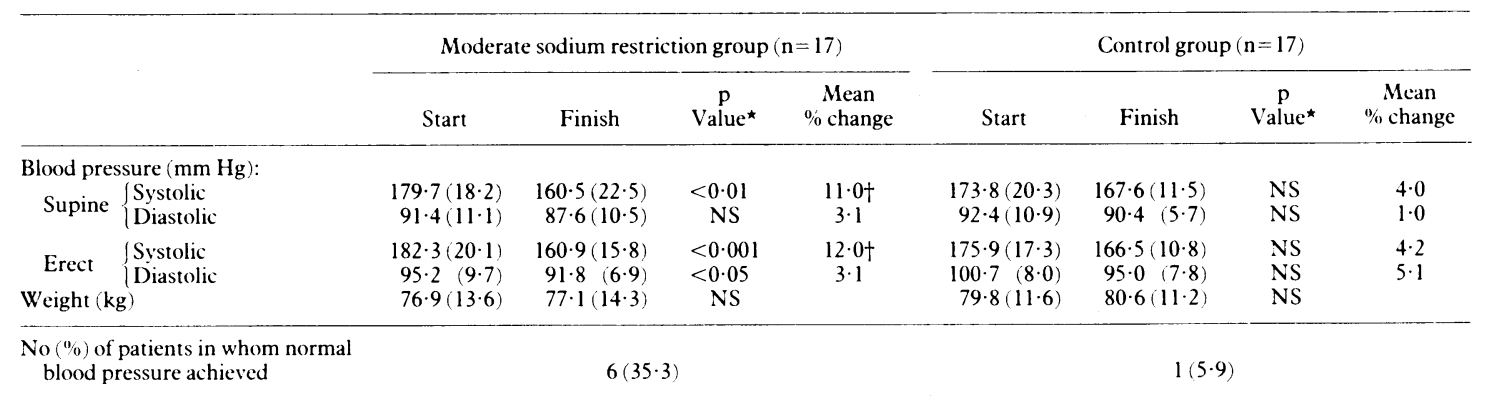

*Paired $t$ test. $\quad$ †Significance of difference from controls $\mathrm{p}<0.03$ (unpaired $t$ test). 


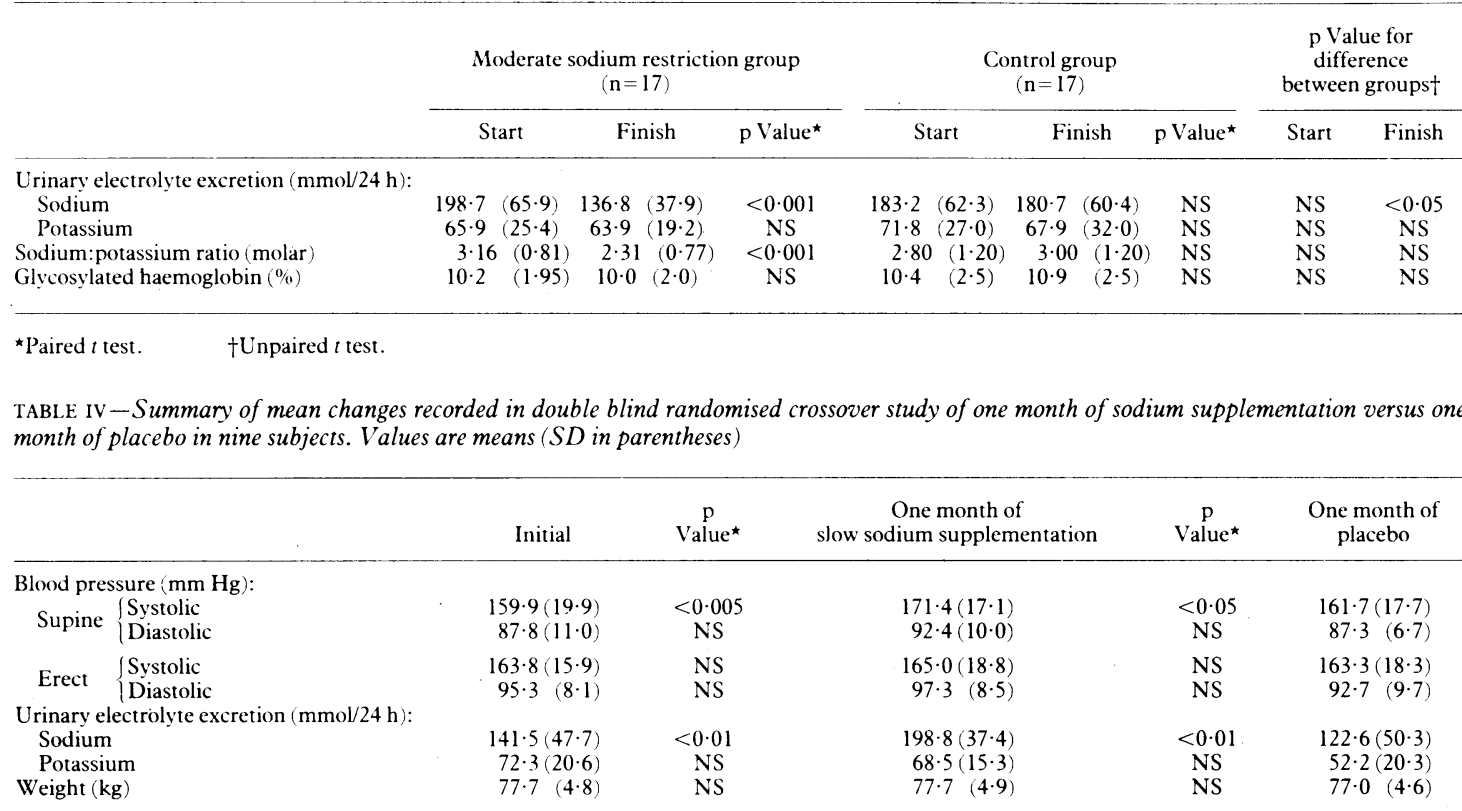

^Paired $t$ test

No significant differences were found between initial and placebo mean values. Five patients received the sodium supplement first, followed by placebo; four patients the reverse. For ease of presentation results are combined for the two groups.

trial. The main finding (table IV) was a significant increase in supine systolic blood pressure with sodium supplementation $(p<0.005)$ and return of supine systolic pressure to near the initial value with placebo. Supine diastolic and erect systolic and diastolic blood pressures showed a similar trend but changes did not reach significance. Analysis of data separately for patients who received the sodium supplement or placebo first showed no order effect, and similar changes were recorded in mean supine systolic pressure in the two groups $(\mathrm{n}=5$ : initial 149.0 (SD $15 \cdot 4) \mathrm{mm} \mathrm{Hg}$, rising to $163.0(14.3) \mathrm{mm} \mathrm{Hg}$ with sodium supplementation $(\mathrm{p}<0.001)$, and returning to $158(14.9) \mathrm{mm} \mathrm{Hg}$ with placebo; $\mathrm{n}=4$ : initial $169 \cdot 5$ (SD 18.7) $\mathrm{mm} \mathrm{Hg}$, falling to $165 \cdot 5(19 \cdot 4) \mathrm{mm} \mathrm{Hg}$ with placebo, and rising to $179 \cdot 8(15 \cdot 7) \mathrm{mm} \mathrm{Hg}$ with sodium supplementation $(\mathbf{p}<0.05))$.

These changes were accompanied by a significant increase in urinary sodium excretion during the sodium supplementation period and a fall to near initial values in the placebo period. No significant changes, however, were noted in mean weight, alcohol consumption, or random blood glucose concentrations.

\section{Discussion}

The important finding of this study was a significant fall in systolic blood pressure with moderate sodium restriction in type II diabetic hypertensive patients. The changes in systolic blood pressure were two to three times greater during sodium restriction but in the parallel study the confidence limits were large. Nevertheless, the finding of a significant rise in systolic pressure with sodium supplementation and a fall with placebo in the randomised crossover study suggests that systolic pressure is sensitive to sodium intake in diabetic hypertensives.

These findings also agree with several controlled studies of similar design. In particular, MacGregor et al reported a small but significant change in both systolic and diastolic blood pressure in a randomised double blind crossover study of sodium supplementation versus placebo in patients with established essential hypertension. ${ }^{13}$ Likewise, in a parallel controlled trial incorporating sodium restriction along with other important dietary changes a relation was shown between reduction of urinary sodium excretion and blood pressure in diabetic hypertensive patients.

No other trial has been designed as a single blind parallel study continuing into a double blind randomised crossover study. This trial was designed to overcome many pitfalls, by combining randomisation and the use of a separate blind observer in combination with recordings on a random zero sphygmomanometer. The lack of response found in diastolic pressure as compared with the change in systolic pressure was interesting but may reflect the numbers of patients. Previous data suggest that sodium restriction should have had an effect on diastolic pressure, and in the double blind crossover study a small rise with sodium supplementation was observed, though the rise did not achieve statistical significance.

A larger number of patients in the sodium restricted group had normal blood pressure at the end of the three month study period, so reducing the number of patients who would otherwise have received antihypertensive drug treatment. This underlines the fact that the fall in systolic blood pressure with sodium restriction should be a clinically useful effect and reflects a worthwhile response, particularly as isolated systolic hypertension is a common problem among type II diabetics. ${ }^{2}$

The exact mechanism of the hypotensive effect of sodium restriction is not known. A possible explanation may lie in the effects on insulin concentrations. Though no direct evidence is available on the effect of sodium intake on insulin secretion, preliminary data suggest that varying sodium content of food may have an influence on glucose response in non-diabetics. ${ }^{14}$

Long term compliance and palatability of this form of dietary approach to the treatment of hypertension are crucial. Data from a study of diabetic hypertensives over one year using a dietary regimen incorporating sodium restriction are encouraging, as urinary sodium excretion remained similar at three months and after one year.$^{15}$ More recent data from small and large trials have also suggested that compliance with simple and moderate advice is achievable over at least four years. ${ }^{16} 17$

The results of this study suggest that moderate sodium restriction in type II diabetic hypertensive 
patients produces a clinically significant reduction in systolic blood pressure. Simple dietary advice on salt intake, similar to that currently recommended for diabetic patients, therefore appears to be appropriate for type II diabetic hypertensives.

We are grateful to Ciba (Horsham) for supplies of Slow Sodium and placebo.

\section{APPENDIX}

Summary of dietary advice given to patients to achieve sodium restriction

Patients were instructed to avoid:

(a) Adding table salt

(b) Adding salt in cooking

(c) Salted meats and smoked fish

(d) Tinned foods - in particular tinned meats, vegetables, fish, and tinned and packeted soups

(e) Salted cheeses

(f) Oxo, Bovril, Marmite, and Bisto

(g) Bottled sauces and savoury snacks, including crisps and peanuts

Controls were instructed to continue with usual eating habits, and no advice was given to either group with regard to carbohydrate, fat, or protein intake.

1 Christlieb AR, Warran JH, Krolewski AS, et al. Hypertension: the major risk factor in juvenile onset insulin dependent diabetics. Diabetes 1981;30 (suppl 2):90-6.

2 Pacy PJ, Dodson PM, Beevers M, Fletcher RF, Taylor KG. The prevalence of hypertension in white, black and Asian diabetics in a district hospita diabetic clinic. Diabetic Medicine 1985;2:125-30.

3 de Chatel R, Weidmann P, Flammer J. Sodium, renin, aldosterone, catecholamines and blood pressure in diabetes mellitus. Kidney Int 1977;12
$412-21$.

4 Weidmann P, Beretta-Picolli C, Keusch G, et al. Sodium-volume factor, cardiovascular reactivity and hypotensive mechanisms of diuretic therapy in mild hypertension associated with diabetes mellitus. $\mathrm{Am}$ J Med 1979;67: 779-84.

5 Roland JM, O'Hare JP, Walters G, Corrall RJM. Sodium retention in response to saline infusion in uncomplicated diabetes mellitus. Diabetes Research 1986;3:213-5.

$6 \mathrm{Ng} \mathrm{LL}$, Hockaday TD. The leucocyte sodium pump in healthy and obese subjects: the association of insulin with its activity. Clin Endocrinol 1986;25:383-92.

7 Dodson PM, Pacy PJ, Bal P, Kubicki AJ, Fletcher RF, Taylor KG. A controlled trial of a high fibre, low fat and low sodium diet for mild hypertension in type 2 (non-insulin dependent) diabetic patients. hypertension in type 2
Diabetologia 1984;27:522-6.

8 De Fronzo RA. The effect of insulin on renal sodium metabolism. Diabetologia 1981;21:165-71.

9 Modan M, Halkin H, Almog S. Hyperinsulinemia: a link between hypertension, obesity and glucose intolerance. $\mathcal{F}$ Clin Invest 1985;75:809-17.

10 Dodson PM, Horton RC. The hypertension of diabetes mellitus: mechanism and implications. Joumal of Human Hypertension 1988;1:241-7.

11 Feldt-Rasmussen B, Mathiesen ER, Deckert T, et al. Central role of sodium in the pathogenesis of blood pressure changes independent of angiotensin, aldosterone and catecholamines in type I (insulin-dependent) diabetes mellitus. Diabetologia 1987;30:610-7.

12 World Health Organisation. Report of the WHO Expert Committee on Hypertension. WHO Tech Rep Ser 1978; No 646.

13 MacGregor GA, Markandu N, Best F, et al. Double blind randomised crossover trial of moderate sodium restriction in essential hypertension. Lancet 1982;i:351-4.

14 Thorburn AW, Brand JC, Truswell AS. Salt and the glycaemic response. BrMed 7 1986;292:1697-9.

15 Dodson PM, Pacy PJ, Webberley MJ, Fletcher RF, Taylor KG. One year follow-up treatment of hypertension in type II diabetic subjects with a high cereal fibre, low fat and low sodium dietary regimen [Abstract]. Proc Nutr Soc 1985; 44:129.

16 Stamler R, Stamler J, Grimm R, et al. Nutritional therapy for high blood pressure. JAMA 1987;257:1484-91

17 Dodson PM, Pacy PJ, Cox EV. Long term follow-up of the treatment of essential hypertension with a high fibre, low fat and low sodium dietary regimen. Hum Nutr Clin Nutr 1985;39C:213-20.

(Accepted 11 November 1988)

\section{Malignant fibrous histiocytoma: a new complication of chronic venous ulceration}

\section{J Berth-Jones, R A C Graham-Brown, A Fletcher, H P Henderson, W W Barrie}

Leicester Royal Infirmary, Leicester LE1 5 WW

J Berth-Jones, MRCP, registrar in dermatology R A C Graham-Brown, MRCP, consultant in dermatology

A Fletcher, MRCPATH, consultant in histopathology H P Henderson, FRCS, consultant in plastic surgery W W Barrie, FRCS, consultant surgeon

Correspondence to: Dr Berth-Jones.

Br.Med J 1989;298:230-1
We report three cases of malignant fibrous histiocytoma as a complication of chronic venous ulceration. This highly malignant sarcoma has not previously been reported in this condition.

\section{Case reports}

Case 1-A 77 year old woman presented in 1981 with an ulcer over the left medial malleolus. The ulcer had been present for six years but had increased in size over the previous 12 months. On examination she had extensive bilateral varicose veins. The pedal pulses were normal. The ulcer had a sloughy base and measured $10 \mathrm{~cm}$ by $7.5 \mathrm{~cm}$, and the edge was slightly thickened. A biopsy specimen showed the histological features of malignant fibrous histiocytoma (see below). Her leg was amputated below the knee. She made an excellent recovery and used a prosthesis. There was no subsequent evidence of any metastasis.

Case 2-An 80 year old woman presented in 1983 with an ulcerated mass over the right medial malleolus. She had a history of chronic venous ulceration of the right leg dating back to a pregnancy in 1931 . Examination showed a fungating tumour over the right medial malleolus surrounded by numerous scars from healed ulcers. Histological examination showed malignant fibrous histiocytoma (see below). Her leg was amputated below the knee, and there was no sign of recurrent disease when she died six months later.

Case 3-An 81 year old woman presented in 1984 with a large ulcer over the medial aspect and front of the right lower leg. This had developed 12 months earlier after she fell and abrased her leg. She had bilateral varicose veins and stasis eczema on the ulcerated leg. After three months of follow up the margins of the ulcer had become raised and haemorrhagic. Biopsy showed the histological features of malignant fibrous histiocytoma (see below). Her leg was amputated below the knee, and a prosthetic limb was fitted, which she used without difficulty. She developed nodes in the groin five months later, which were thought clinically to be malignant. These were treated with radiotherapy, and there was no further evidence of metastasis.

Histological findings-All three tumours showed pleomorphic spindle cells with occasional multinucleate cells and mitotic activity ranging from one to four mitoses in each high power field (see figure). A storiform pattern was present in cases 1 and 3 but was less pronounced in case 2. Areas of necrosis and haemorrhage were present in case 1 . In all three cases malignant fibrous histiocytoma was diagnosed

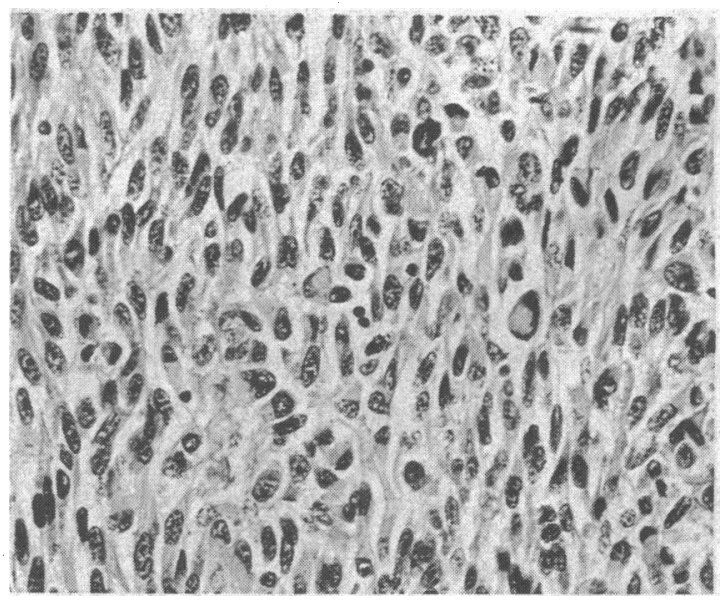

Pleomorphic spindle cells arranged haphazardly in malignant fibrous histiocytoma from case 3 . Stained with haematoxylin and eosin; $\times 500$ 